\title{
Development and validation of an algorithm to estimate eplet mismatch by substituting imputation of HLA antigens for high- resolution HLA typing based on Korean HLA frequencies
}

\footnotetext{
'Department of Laboratory Medicine, Ewha Womans University Mokdong Hospital, Seoul, Korea

${ }^{2}$ Department of Laboratory Medicine, Korea University Guro Hospital, Seoul, Korea

${ }^{3}$ Department of Internal Medicine, Seoul National University Bundang Hospital, Seongnam, Korea
} \\ ${ }^{4}$ Department of Laboratory Medicine, Seoul National University Bundang Hospital, Seongnam, Korea \\ ${ }^{5}$ Department of Internal Medicine, Seoul National University Hospital, Seoul, Korea
}

Soo-Kyung Kim ${ }^{1}$, Borae G. Park ${ }^{2}$, Hyung Eun Son ${ }^{3}$, Jong Cheol Jeong ${ }^{3}$, Yun Ji Hong ${ }^{4}$, Dong Wan Chae ${ }^{5}$

Background: Assessment of eplet mismatches in transplantation requires high-resolution (HR) molecular human leukocyte antigen (HLA) typing. However, HR typing is rarely applied to solid organ transplantation. We developed an algorithm to derive an HR genotype from a low-resolution (LR) one and evaluated its performance in a Korean population.

Methods: Our multistep imputation process is based on an HLA haplotype and allelic frequency dataset for the Korean population. To evaluate the performance of the algorithm, the HR typing results of 127 Korean patients who underwent sequence-based typing (SBT) were converted to LR HLA-A, -B, and DR genotypes. HR genotypes were then derived from the LR genotypes using the algorithm. We compared the HR genotypes derived using the algorithm with those determined by SBT.

Results: A total of $94.4 \%(120 / 127)$ of the LR genotypes could be converted into HR genotypes using the algorithm. Also, 59 (46.5\%) could be assigned an HR genotype based on haplotype frequencies for the Korean population. There were 28 errors (23.7\%) in A, $12(10.2 \%)$ in $B$, and $23(19.5 \%)$ in DR. Additionally, for the $61 \mathrm{HR}$ genotypes (48.0\%) assigned using our algorithm, and there were 11 errors (9.0\%) in $A, 10(8.2 \%)$ in $B$, and 28 (23.0\%) in DR. Overall, the HR genotype was inaccurate for $16.3 \%$ (39/240) of HLA-A, 9.2\% (22/240) of HLA-B, and 21.3\% (51/240) of HLA-DR. In particular, A2 ( $n=30)$ and A26 ( $n=6)$ of HLA-A, B62 $(n=8)$ and B61 $(n=7)$ of HLA-B, and DR4 $(n=23)$, DR14 $(n=8)$, and DR15 $(n=6)$ of HLA-DR showed high error rates. According to the differences in HR genotypes, the eplet differences were up to four in $A$, three in $B$, and seven in DR.

Conclusions: The error rate of our algorithm was lower than that previously applied to non-Caucasian populations. However, it should be used cautiously because it can yield erroneous results.

Corresponding author: Borae G. Park

E-mail: borae.park@gmail.com

\section{(c) The Korean Society for Transplantation}

This is an Open Access article distributed under the terms of the Creative Commons Attribution Non-Commercial License (http://creativecommons.org/licenses/by-nc/4.0/) which permits unrestricted non-commercial use, distribution, and reproduction in any medium, provided the original work is properly cited. 